\title{
Efficacy and Safety of Baricitinib in Japanese Patients with Active Rheumatoid Arthritis Receiving Background Methotrexate Therapy: A 12-week, Double-blind, Randomized Placebo-controlled Study
}

\author{
Yoshiya Tanaka, Kahaku Emoto, Zhihong Cai, Takehiro Aoki, Douglas Schlichting, \\ Terence Rooney, and William Macias
}

\begin{abstract}
Objective. To evaluate efficacy and safety, baricitinib [Janus kinase (JAK) 1/JAK2 inhibitor] was compared with placebo in Japanese patients with active rheumatoid arthritis (RA) despite background treatment with methotrexate (MTX).

Methods. This was a phase IIB, double-blind, randomized, placebo-controlled study (clinicaltrials.gov: NCT01469013). Patients had moderate to severe active adult-onset RA despite stable treatment with MTX. Patients $(\mathrm{n}=145)$ were randomized in a 2:1:1:1:1 ratio to placebo or $1 \mathrm{mg}, 2 \mathrm{mg}, 4 \mathrm{mg}$, or 8 $\mathrm{mg}$ oral baricitinib daily for 12 weeks. The primary analysis compared the combined 4/8-mg dose groups with placebo for the American College of Rheumatology (ACR) 20 response rate at 12 weeks Other outcomes included additional measures of disease activity, physical function, laboratory abnormalities, and adverse events.

Results. A significantly higher proportion of patients in the combined 4/8-mg baricitinib group (37/48, $77 \%$ ) compared with the placebo group $(15 / 49,31 \%)$ had at least an ACR20 response after 12 weeks of treatment $(\mathrm{p}<0.001)$. Significant improvements in disease activity, remission, and physical function were observed as early as Week 2 of treatment with baricitinib, particularly with daily doses of $\geq 4$ $\mathrm{mg}$. Only 1 patient receiving baricitinib discontinued because of an adverse event. Adverse event rates with baricitinib doses $\leq 4 \mathrm{mg}$ daily were similar to placebo, but there was a higher incidence of adverse events and laboratory abnormalities in the $8-\mathrm{mg}$ group.

Conclusion. In this phase II study, baricitinib was well tolerated and rapidly improved the signs, symptoms, and physical function of Japanese patients with active RA, supporting continued development of baricitinib (clinicaltrials.gov NCT01469013). (First Release February 1 2016; J Rheumatol 2016;43:504-11; doi:10.3899/jrheum.150613)
\end{abstract}

Key Indexing Terms:

\section{RHEUMATOID ARTHRITIS}

Proinflammatory cytokines implicated in the pathogenesis of rheumatoid arthritis (RA), including interleukin (IL) 6, IL-15, IL-17, IL-23, interferon- $\alpha / \beta$, interferon- $\gamma$, and granulocyte-macrophage colony stimulating factor ${ }^{1,2}$, primarily act through intracellular Janus kinase (JAK) signaling pathways ${ }^{3}$. Drugs that inhibit these pathways ${ }^{3,4}$, which may directly block cytokine signaling or indirectly modulate $\mathrm{T}$ cell functions through suppression of CD80/86 expression in

From the First Department of Internal Medicine, School of Medicine, University of Occupational and Environmental Health, Kitakyushu; Eli Lilly and Co., Tokyo; Eli Lilly Japan K.K., Kobe, Japan; Eli Lilly and Co., Indianapolis, Indiana, USA.

Sponsored by Eli Lilly Japan K.K. and Eli Lilly and Co., manufacturer/licensee of baricitinib. Eli Lilly Japan K.K. and Eli Lilly and Co. were involved in the study design, data collection, data analysis, and preparation of the manuscript. Medical writing assistance was provided by Janelle Keys, PhD, CMPP, and Rebecca Lew, PhD, CMPP, of ProScribe, part of the Envision Pharma Group, and was funded by Eli Lilly Japan K.K. ProScribe's services complied with international guidelines for Good Publication Practice (GPP2). ZC and TA are employees of Eli Lilly Japan K.K. KE, DS, TR, and WM are employees of Eli Lilly and Co. and KE owns stock in the company.

\section{BARICITINIB JAPAN}

dendritic cells 5 , are currently the focus of extensive development efforts for RA. Baricitinib is a potent, selective, orally administered, reversible inhibitor of JAK signaling ${ }^{6}$. In contrast to other JAK inhibitors ${ }^{7,8}$, baricitinib has similar inhibitory activity for both JAK1 [50\% Inhibitory Concentration (IC50) $5.9 \mathrm{nM}$ ] and JAK2 (IC50 $5.7 \mathrm{nM}$ ), but much less activity against JAK3 (IC50 > $400 \mathrm{nM}$ ) and tyrosine kinase 2 (IC50 $53 \mathrm{nM})^{6}$ in isolated enzyme assays.

\footnotetext{
Y. Tanaka, MD, PhD, Professor, First Department of Internal Medicine, School of Medicine, University of Occupational and Environmental Health; K. Emoto, MD, PhD, Medical Advisor, Eli Lilly and Co.; Z. Cai, PhD, Senior Associate, Eli Lilly Japan K.K.; T. Aoki, PhD, Senior Associate, Eli Lilly Japan K.K.; D. Schlichting, RN, MS, Principal Research Scientist, Eli Lilly and Co.; T. Rooney, MD, Medical Director, Eli Lilly and Co.; W. Macias, MD, PhD, Distinguished Medical Fellow, Eli Lilly and Co.

Address correspondence to Dr. K. Emoto, Eli Lilly and Co., Akasaka, Minato-ku,Tokyo, 107-0052, Japan.E-mail: emoto_kahaku@lilly.com Full Release Article. For details see Reprints/Permissions at jrheum.org Accepted for publication November 13, 2015.
} 
In a multinational, phase IIB randomized study, baricitinib was effective and well tolerated in patients $(\mathrm{n}=301)$ who had active RA despite methotrexate (MTX) therapy ${ }^{9}$. After 12 weeks, significantly more patients in the combined 4/8-mg daily baricitinib dose group achieved an American College of Rheumatology (ACR) 20 response compared with placebo ( $76 \%$ vs $41 \%, \mathrm{p}<0.001$ ). Adverse event rates were similar in the combined 4/8-mg baricitinib group and the placebo group. In the open-label extension of the study, clinical improvements observed with baricitinib were maintained through 128 weeks and safety signals were consistent with those seen in the first 12 weeks of treatment ${ }^{10,11}$.

Asian and non-Asian populations differ in genetic background ${ }^{12}$, RA prevalence ${ }^{13}$, and demographic characteristics. In addition, in clinical practice, patients with RA in Japan are often prescribed lower doses of MTX $(<9$ mg per week in average doses ${ }^{14,15}$, until recently) compared with patients in the United States (>15 mg per week ${ }^{16}$ ), which may theoretically affect the response to, and tolerability of, concomitant RA medication. Therefore, investigating the efficacy and safety of baricitinib in an Asian population is important before initiating phase III studies. The primary objective of our phase II study was to evaluate the efficacy (by ACR20 response rate) of baricitinib [combined 4/8-mg once daily (QD) groups] compared with placebo after 12 weeks of treatment in Japanese patients with active RA despite background MTX therapy.

\section{MATERIALS AND METHODS}

Study design. This was a phase II, randomized, placebo-controlled study (clinicaltrials.gov NCT01469013) with a 12-week, double-blind treatment period followed by a 52-week, single-blind extension treatment period. Our study was conducted at 24 sites in Japan from November 2011 to December 2013, with an interim lock date for the double-blind treatment period in December 2012. Our study was approved by the institutional review board or ethics committee at each site and was conducted in accordance with the Declaration of Helsinki, Good Clinical Practice guidelines, and all applicable laws and regulations in Japan. All participants were outpatients who provided written informed consent.

Study population. Male and female patients were included who were aged 20 to 75 years inclusive, with a diagnosis of adult-onset RA (of functional Class I, II, or $\mathrm{III}^{17}$ ), according to the $2010 \mathrm{ACR} /$ European League Against Rheumatism (EULAR) classification criteria ${ }^{18}$. Disease duration of at least 6 months and at most 15 years was required. Patients had active RA, defined as having at least 6 swollen and at least 6 tender joints based on the 66 and 68 joint counts, respectively ${ }^{19}$, and a C-reactive protein (CRP) measurement $>0.5 \mathrm{mg} / \mathrm{dl}$ or an erythrocyte sedimentation rate $(\mathrm{ESR})>28 \mathrm{~mm} / \mathrm{h}$. For patients receiving corticosteroids, the dose must have been stable with no dose adjustment allowed during the 12-week study period and must not have exceeded $10 \mathrm{mg}$ prednisone (or equivalent) daily. Patients must have regularly received MTX for at least 12 weeks and at a stable dose (6 to 16 $\mathrm{mg} /$ week) for at least 8 weeks before randomization.

The main exclusion criteria for the study population were the use of conventional disease-modifying antirheumatic drugs other than MTX and/or sulfasalazine in the 8 weeks before randomization, prior biological therapy discontinued because of insufficient efficacy, and significant hematological or chemical abnormalities identified during screening [hemoglobin $<10.0$ $\mathrm{g} / \mathrm{dl}$, total platelet count $<100,000 / \mu 1$, total white blood cell count $<2500 / \mu 1$, neutrophil count $\leq 1200 / \mu 1$, lymphocyte count $\leq 750$ cells $/ \mu 1$, and aspartate aminotransferase (AST) or alanine aminotransferase (ALT) concentrations $>3$ times the upper limit of normal].

Treatment protocol. Patients were randomized (by a computer-generated random sequence using an interactive voice response system) in a 2:1:1:1:1 ratio to placebo or $1 \mathrm{mg}, 2 \mathrm{mg}, 4 \mathrm{mg}$, or $8 \mathrm{mg}$ QD oral baricitinib (LY3009104, Eli Lilly and Co., formerly INCB028050, Incyte Corp.). The duration of the double-blind treatment period was 12 weeks, and efficacy and safety assessments were conducted at baseline, Week 2, Week 4, Week 8 , and Week 12 (primary efficacy endpoint). The double-blind treatment period was followed by a 52-week extension treatment period, with patients receiving placebo, 1 or $2 \mathrm{mg}$ baricitinib blindly rerandomized (1:1) to $4 \mathrm{mg}$ or $8 \mathrm{mg}$ oral baricitinib daily, and patients receiving 4 or $8 \mathrm{mg}$ baricitinib remaining on the same dose. The results of the extension treatment period will be reported separately.

Efficacy measures. Relief of signs and symptoms of RA was assessed using the ACR20 responder index ${ }^{20}$. Other efficacy outcomes included the following: ACR50 and ACR70 responses; ACR core components [tender joint count, swollen joint count, physician's global assessment of disease activity, patient's global assessment of disease activity, patient's assessment of arthritis pain, the Health Assessment Questionnaire-Disability Index (HAQ-DI), and high-sensitivity CRP (hsCRP)]; Disease Activity Score (DAS), reported as DAS28-CRP and DAS28-ESR; Simplified Disease Activity Index (SDAI); and the EULAR28 response criteria, categorizing patients as nonresponders, moderate responders, or good responders. Low disease activity was defined as DAS28-CRP or DAS28-ESR $\leq 3.2$ and SDAI $\leq 11.0$, and remission was defined as DAS28-CRP or DAS28-ESR $<2.6$ and $\mathrm{SDAI} \leq 3.3$. A minimal clinically important difference (MCID) in HAQ-DI was defined as a change from baseline of $\geq-0.22$. Efficacy measures were assessed by the proportion of patients who met the predefined criteria for response or score, or by the change over time.

Safety measures. Treatment-emergent adverse events (TEAE) were coded and summarized using the Medical Dictionary for Regulatory Activities, Version 15.1 and Version 16.1, and laboratory measures were assessed.

Statistical analysis. The sample size was estimated to provide $80 \%$ power to detect a $25 \%$ difference at Week 12 in ACR20 response between the combined 4/8-mg baricitinib group and the placebo group (assuming a placebo response rate of $30 \%$ ). Based on this assumption, 24 patients per dose of baricitinib were required to compare with 48 patients receiving placebo using a 1-sided chi-square test at the 0.05 level of significance.

The result from the combined 4/8-mg baricitinib groups was assessed as the primary comparison for ACR20 response rate compared with placebo because previous studies have demonstrated that doses of $4 \mathrm{mg}$ or greater achieved a similar and near-maximum efficacy response after 12 weeks of treatment $^{9,21}$. The 1-mg and 2-mg doses were included to define the lower end of the dose range.

All randomized patients were included in the efficacy and safety analyses. The primary efficacy analysis used a logistic regression model that included the treatment group (combined 4/8-mg baricitinib or placebo) as a fixed factor and baseline DAS28-CRP as a continuous covariate. Tests for treatment effects were also conducted on binary measures using the Fisher's exact test at a 1 -sided $\alpha$ level of 0.05 , and on continuous measures using an ANCOVA model that included the dose group as a fixed factor and the baseline value of the measure as a continuous covariate at a 2 -sided $\alpha$ level of 0.05 . Dose response was assessed using a 1-sided test ( $\alpha$ level of 0.05 ) of the Cochran-Armitage trend test for the binary measures, and a 2-sided test ( $\alpha$ level of 0.05 ) of the Spearman nonparametric correlation analysis for continuous measures. Patients who dropped out of the study were treated as nonresponders (for ACR20, ACR50, and ACR70) from the time of discontinuation. Demographic and baseline characteristics were summarized using descriptive statistics. Analyses were performed using SAS Version 9.2 (SAS Institute Inc.).

\section{RESULTS}

Patient characteristics and disposition. There were 199

Personal non-commercial use only. The Journal of Rheumatology Copyright (c) 2016. All rights reserved. 
patients screened for inclusion in our study. Of these, 145 patients were randomized to treatment and received at least 1 dose of study drug (Figure 1). Of the 54 patients not randomized, 1 withdrew and 53 did not meet eligibility criteria for reasons including hematological abnormalities $(\mathrm{n}=24)$, positive tuberculosis test result $(\mathrm{n}=15)$, and low hsCRP $(\leq 0.5 \mathrm{mg} / \mathrm{dl})$ or low ESR $(\leq 28 \mathrm{~mm} / \mathrm{h} ; \mathrm{n}=6)$.

The majority of patients enrolled in the study were women who were positive for both anticyclic citrullinated peptide and rheumatoid factor. Mean age was 53.6 years, mean duration of RA was 5.67 years, and mean dose of MTX was $8.7 \mathrm{mg} /$ week (Table 1). Baseline demographics and measures of disease activity were similar across the treatment groups, except for HAQ-DI, which was lower in the 8-mg group (Table 1).

At Week 12, 142 patients remained on treatment and completed the final visit (Figure 1). The reasons for early discontinuation from the study were adverse events $(n=2)$ and patient decision $(\mathrm{n}=1)$.

ACR responses. There were significantly more patients in the combined 4/8-mg baricitinib group $(37 / 48,77 \%)$ compared with the placebo group $(15 / 49,31 \%)$ who had an ACR20 response after 12 weeks of treatment $(\mathrm{p}<0.001)$.

For the 4-mg and 8-mg baricitinib groups, the proportion of patients with an ACR20 response was similar between doses and significantly greater than placebo (Figure 2, top panel). A treatment benefit was observed from as early as 2 weeks (the first scheduled postbaseline visit) after the start of treatment, and near-maximal treatment effects were observed by 8 weeks of treatment (Figure 2, second panel). For the 1-mg and 2- $\mathrm{mg}$ baricitinib groups, the proportion of patients with an ACR20 response was also significantly greater than placebo (Figure 2, top panel), although the onset of treatment response was not as rapid as that observed with the higher doses of baricitinib (Figure 2, second panel).

Compared with the placebo group, there was a significantly greater proportion of patients in all baricitinib dose groups who had an ACR50 or ACR70 response at Week 12 (Figure 2, top panel). Baricitinib treatment improved all ACR core components compared with placebo. For most components, baricitinib doses of $4 \mathrm{mg}$ and $8 \mathrm{mg}$ daily provided a more rapid onset of benefit than lower doses (Supplementary Figures available online at jrheum.org). For all ACR core components, there was also a statistically significant baricitinib dose response $(\mathrm{p}<0.001)$ at 12 weeks of treatment, although similar efficacy was observed for some measures for doses of $2 \mathrm{mg}$ and greater at Week 12.

Other efficacy outcomes. Disease activity was improved by all doses of baricitinib across numerous additional composite efficacy outcome measures (Figure 3). For most measures, response rates were higher for the 4-mg and 8-mg baricitinib groups than in the 1-mg and 2-mg baricitinib groups, reflective of the dose response.

Summary of TEAE. Baricitinib was well tolerated during the study. All baricitinib groups, apart from the 8-mg group, reported an incidence of TEAE similar to the placebo group (Table 2). Common TEAE reported in both the placebo and baricitinib groups with similar incidence included nasopharyngitis, increased blood creatine phosphokinase (CPK), pharyngitis, hyperlipidemia, and lymphopenia. Abnormal hepatic function, abnormal liver function test, and hypercholesterolemia were reported only in patients treated with baric-

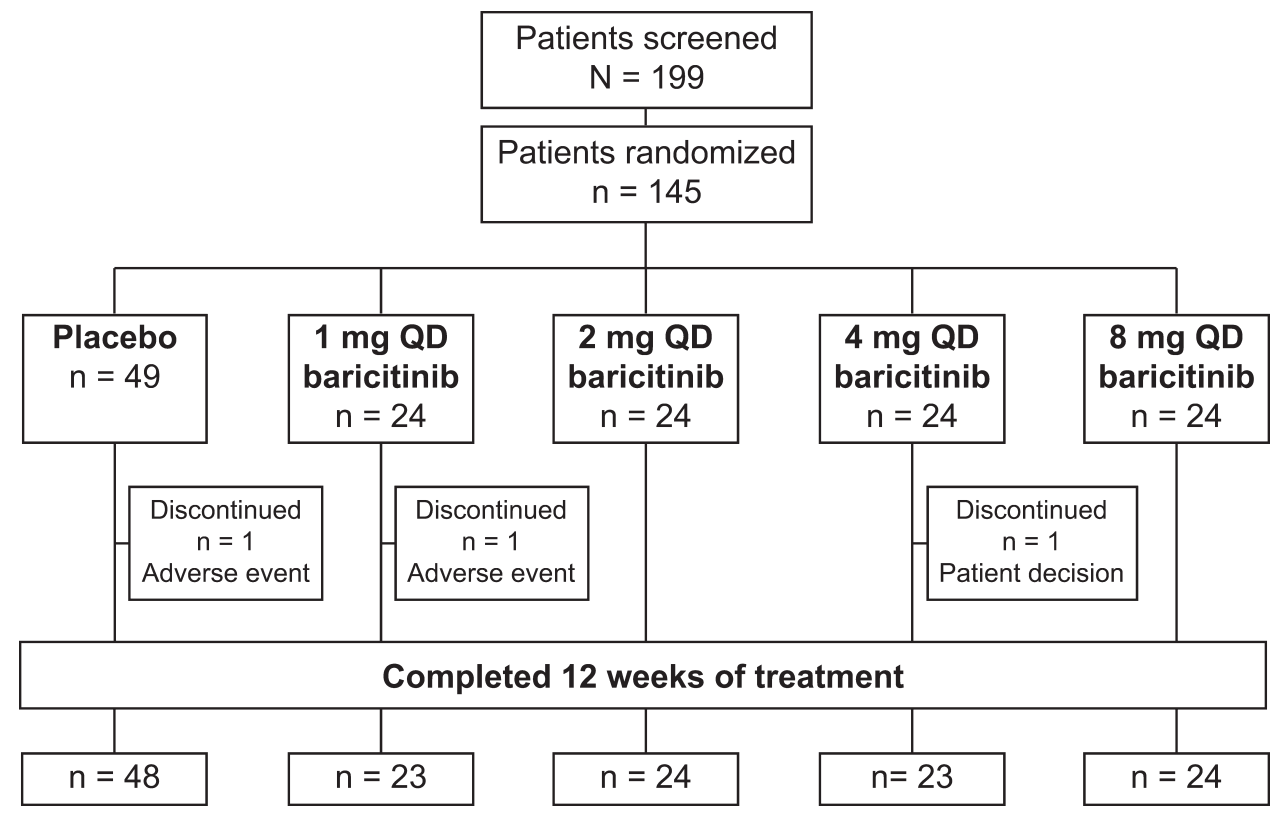

Figure 1. Patient flow diagram for the 12-week study of Japanese patients with rheumatoid arthritis treated with baricitinib or placebo. QD: once daily. 
Table 1. Patient demographics and characteristics at baseline for Japanese patients $(\mathrm{n}=145)$ with RA. Values are mean (SD) unless otherwise specified.

\begin{tabular}{|c|c|c|c|c|c|}
\hline \multirow[t]{2}{*}{ Characteristics } & \multirow[t]{2}{*}{ Placebo, $n=49$} & \multicolumn{4}{|c|}{ Baricitinib QD } \\
\hline & & $1 \mathrm{mg}, \mathrm{n}=24$ & $2 \mathrm{mg}, \mathrm{n}=24$ & $4 \mathrm{mg}, \mathrm{n}=24$ & $8 \mathrm{mg}, \mathrm{n}=24$ \\
\hline Age, yrs & $51.1(12.0)$ & $52.7(12.8)$ & $56.1(11.5)$ & $57.5(10.4)$ & $53.6(11.3)$ \\
\hline Duration of RA, yrs & $5.06(3.96)$ & $6.22(3.27)$ & $6.32(4.18)$ & $5.86(3.95)$ & $5.55(4.62)$ \\
\hline Anti-CCP-positive, \% (n) & $76(37)$ & $79(19)$ & $83(20)$ & $92(22)$ & $79(19)$ \\
\hline RF-positive, \% (n) & $86(42)$ & $75(18)$ & $79(19)$ & $83(20)$ & $79(19)$ \\
\hline Patient's assessment of arthritis pain score* & * $\quad 41.4(28.3)$ & $46.6(26.2)$ & $52.2(24.8)$ & $54.3(21.2)$ & $41.8(20.9)$ \\
\hline Patient's global assessment of DAS* & $42.6(24.6)$ & $49.2(29.4)$ & $52.2(24.8)$ & $49.5(23.2)$ & $37.7(23.7)$ \\
\hline Physician's global assessment of DAS* & $51.0(17.8)$ & $48.2(16.3)$ & $55.3(16.9)$ & $56.6(17.3)$ & $50.2(14.2)$ \\
\hline DAS28-CRP** & $4.72(1.00)$ & $4.60(0.77)$ & $4.94(0.83)$ & $4.96(0.80)$ & $4.67(0.71)$ \\
\hline DAS28-ESR $* *$ & $5.53(0.98)$ & $5.51(0.75)$ & $5.78(0.82)$ & $5.77(0.68)$ & $5.47(0.74)$ \\
\hline SDAI $* * *$ & $27.85(10.52)$ & $25.10(7.86)$ & $29.23(9.83)$ & $30.81(9.72)$ & $27.58(7.81)$ \\
\hline Current use of oral corticosteroid, \% (n) & $59(29)$ & $50(12)$ & $54(13)$ & $75(18)$ & $54(13)$ \\
\hline Dose of corticosteroid, mg/week & $22.9(9.96)$ & $26.8(16.51)$ & $38.0(19.00)$ & $33.6(19.47)$ & $34.7(21.83)$ \\
\hline
\end{tabular}

* Range of scale 0 to $100 . * *$ Range of scale 0 to $9.4 . * * *$ Range of scale 0 to $86 .{ }^{\dagger}$ Range of scale 0 to $3 .{ }^{\ddagger}$ Prednisolone-equivalent dose. RA: rheumatoid arthritis; QD: once daily; anti-CCP: anticyclic citrullinated peptide antibodies; RF: rheumatoid factor; TJC68: tender joint count at 68 joints; SJC66: swollen joint count at 66 joints; DAS28: Disease Activity Score at 28 joints; CRP: C-reactive protein; ESR: erythrocyte sedimentation rate; SDAI: Simplified Disease Activity Index; HAQ-DI: Health Assessment Questionnaire-Disability Index; hsCRP: high-sensitivity CRP; MTX: methotrexate.

itinib, with many of these events reported in the 8-mg baricitinib group. The events of abnormal hepatic function and abnormal liver function tests were transient in character and only 3 events required temporary interruption of study drug administration $(\mathrm{n}=1$ event each for 2-mg, 4-mg, and 8-mg baricitinib groups). There were 2 TEAE that resulted in early discontinuation from the study: pollakiuria in the placebo group, and herpes simplex in the 1-mg baricitinib group (Table 2). The latter event was mild in severity, but the event itself was a discontinuation criterion of the protocol.

There were 3 serious adverse events (SAE) reported (cholecystitis in the placebo group, acute pancreatitis in the 2-mg baricitinib group, and cataract in the 8-mg baricitinib group). All 3 patients completed the study. All TEAE were mild or moderate, except for the event of acute pancreatitis reported as an SAE in the 2-mg baricitinib group. There were no deaths, malignancies, or gastrointestinal perforations reported.

Incidence of infections. Each treatment group reported a similar incidence of infections (Table 2). The most common adverse events related to infection were nasopharyngitis and pharyngitis (Table 2). No serious infection and no tuberculosis, Pneumocystis jirovecii pneumonia, or herpes zoster cases were reported.

Laboratory abnormalities. Changes in a number of laboratory measures were observed following treatment with baricitinib (Table 3; Supplementary Figures available online at jrheum.org). Compared with placebo, baricitinib treatment was associated with small increases in the median concentration of creatinine, ALT, and AST, with larger increases in high-density lipoprotein (HDL) cholesterol, low-density lipoprotein (LDL) cholesterol, and CPK. There was no statistically significant effect on hemoglobin levels. Neutrophil counts were decreased in all treatment groups; the change from baseline was significantly greater for $2 \mathrm{mg}, 4 \mathrm{mg}$, and $8 \mathrm{mg}$ baricitinib compared with placebo. These laboratory changes did not result in discontinuation of patients from our study.

Changes in laboratory measures following treatment were also assessed by Common Terminology Criteria for Adverse Events (CTCAE) grade (Supplementary Table 1 available online at jrheum.org). Grade 1 or higher abnormalities were common at baseline in all treatment groups for hemoglobin concentration, lymphocyte count, and LDL concentration.

For hemoglobin concentration, the percentage of patients experiencing an increase in CTCAE grade on at least 1 occasion was $31 \%, 38 \%, 33 \%, 25 \%$, and $46 \%$ for the placebo, 1-mg, 2-mg, 4- mg, and 8-mg baricitinib groups, respectively.

For lymphocyte count, the percentage of patients experiencing an increase in CTCAE grade on at least 1 occasion was $29 \%, 25 \%, 25 \%, 54 \%$, and $38 \%$ of patients in the placebo, 1-mg, 2-mg, 4-mg, and 8-mg baricitinib groups, respectively. Four patients experienced Grade 3 decreases in lymphocyte count $(\mathrm{n}=1$ in the $2-\mathrm{mg}$ baricitinib group, $\mathrm{n}=$ 2 in the 4-mg group, and $n=1$ in the 8-mg group). In 2 of these 4 patients, the lymphocyte count was abnormal at baseline (Grade 1 and Grade 2 abnormalities). In the remaining 2 patients, the lymphocyte count returned to Grade 2 or less at the next visit (1 patient with and 1 patient without drug interruption).

Personal non-commercial use only. The Journal of Rheumatology Copyright $\odot$ 2016. All rights reserved 

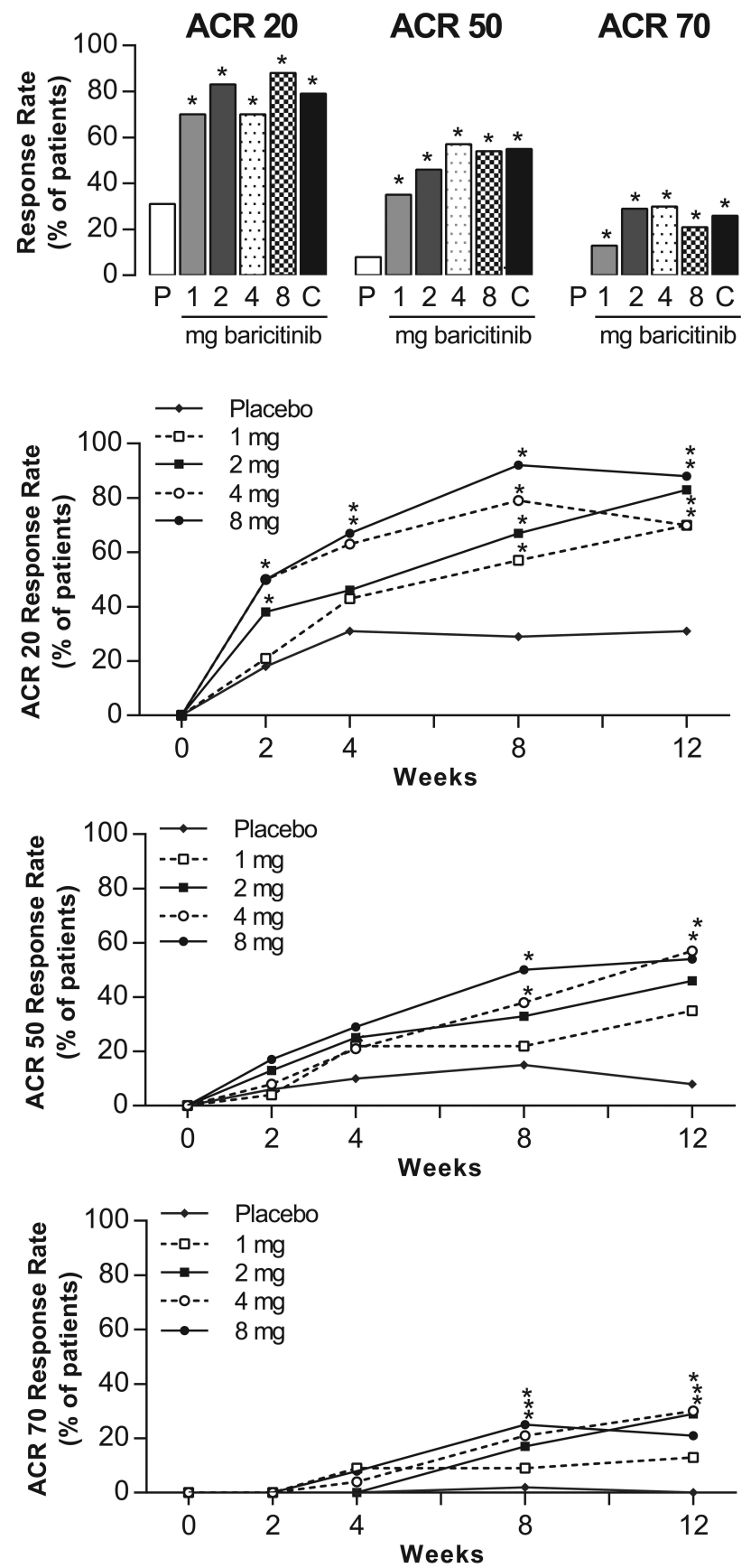

Figure 2. ACR20, ACR50, and ACR70 response rates during the 12-week study of Japanese patients with rheumatoid arthritis treated with baricitinib or placebo. Top panel: Proportion of patients with an ACR20, ACR50, or ACR70 response at 12 weeks of treatment. Bottom panels: Proportion of patients with an ACR20, ACR50, or ACR70 response at 2, 4, 8, and 12 weeks of treatment. Statistical comparisons were based on the Fisher's exact test of each dose versus placebo. $* \mathrm{p}<0.05$. ACR: American College of Rheumatology; C: combined $4 \mathrm{mg}+8 \mathrm{mg}$ baricitinib group; P: placebo.

For ALT concentration, the percentage of patients experiencing an increase in CTCAE grade on at least 1 occasion was $20 \%, 17 \%, 17 \%, 33 \%$, and $29 \%$ for the placebo, $1-\mathrm{mg}$, 2-mg, 4-mg, and 8-mg baricitinib groups, respectively. Three patients experienced a Grade 3 abnormality in ALT concen- tration ( $\mathrm{n}=1$ each for the 2-mg, 4-mg, and 8-mg baricitinib groups). In 2 of these 3 patients, the ALT concentration was abnormal at baseline (Grade 1 and Grade 2 abnormalities). The ALT elevations in these 3 patients were transient and improved by temporary interruption of the study drug, with no recurrence of Grade 3 ALT elevation upon restarting the study drug.

For LDL concentration, $10 \%, 17 \%, 30 \%, 32 \%$, and $25 \%$ of patients in the placebo, 1-mg, 2-mg, 4-mg, and 8-mg baricitinib groups, respectively, who had a baseline LDL concentration of $<130 \mathrm{mg} / \mathrm{dl}$ (no grade or Grade 1) had a value of $\geq 130 \mathrm{mg} / \mathrm{dl}$ (Grade 2 or higher) on at least 1 postbaseline occasion.

There were no reports of Grade 3 or 4 neutropenia; Grade 2, 3, or 4 thrombocytopenia; Grade 3 or 4 elevations in CPK; or Grade 2,3, or 4 elevations in creatinine.

\section{DISCUSSION}

Baricitinib is an oral, reversible inhibitor of the JAK signaling pathway in development for treatment of patients with moderate to severe active RA. To our knowledge, our phase II study is the first randomized placebo-controlled trial to assess baricitinib in Japanese patients. The study population had active disease at baseline, despite established background MTX therapy. Baricitinib at all doses studied was effective at reducing the signs and symptoms of RA. A dose response (across $1 \mathrm{mg}, 2 \mathrm{mg}, 4 \mathrm{mg}$, and $8 \mathrm{mg}$ daily) with increasing efficacy at higher doses was observed for some measures (for example, DAS28-CRP low disease activity, DAS28-CRP remission, EULAR28 moderate or good response), but not for other measures (for example, some ACR response rates, DAS28-ESR). The 4-mg and 8-mg doses of baricitinib were associated with a higher percentage of patients achieving an MCID improvement in HAQ-DI compared with lower doses or placebo. Improvements in disease activity were seen as early as 2 weeks after commencing treatment. Similar effects were observed for the 4-mg and 8-mg doses of baricitinib across most efficacy measures. These findings are consistent with previous studies of baricitinib in non-Japanese patients ${ }^{9,21}$.

The safety profile was consistent with previous studies of baricitinib in non-Japanese patients ${ }^{9,21}$. Baricitinib was generally well tolerated and few patients discontinued the study. The incidence and characteristics of TEAE and SAE, including TEAE related to infection, were generally similar between baricitinib and placebo (with a higher incidence of TEAE in the 8-mg baricitinib group). No malignancies, serious infections, tuberculosis, pneumocystis pneumonia, herpes zoster cases, or gastrointestinal perforations were observed.

Changes in laboratory measures were observed with baricitinib treatment; the type and magnitude of the changes were similar to those seen with baricitinib in non-Japanese patients $^{9}$ and with other JAK inhibitors ${ }^{22}$. Changes in hemoglobin concentrations are of particular interest when evaluating the safety profile of JAK inhibitors with selectivity for JAK2 because erythropoietin signals through a

Personal non-commercial use only. The Journal of Rheumatology Copyright $($ C 2016. All rights reserved. 


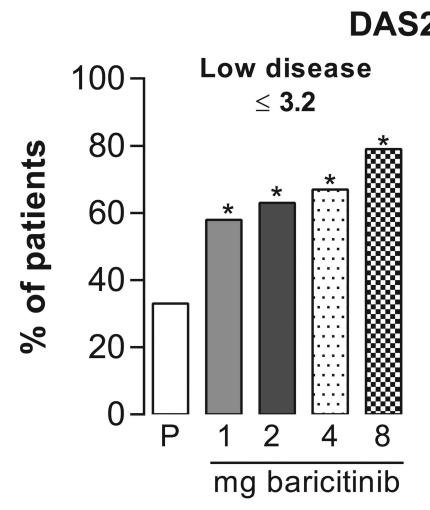

DAS28-CRP

Remission

$<2.6$
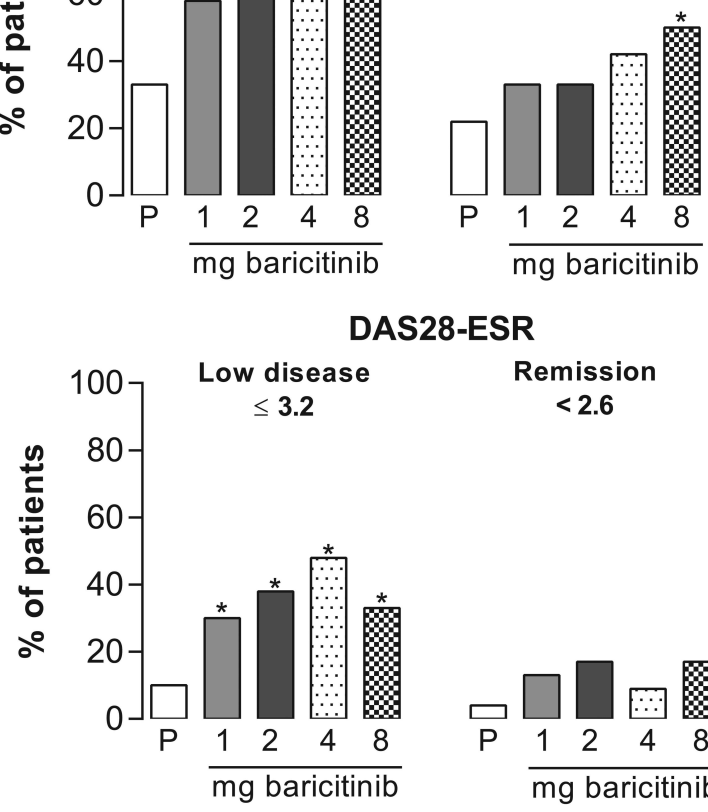

DAS28-ESR

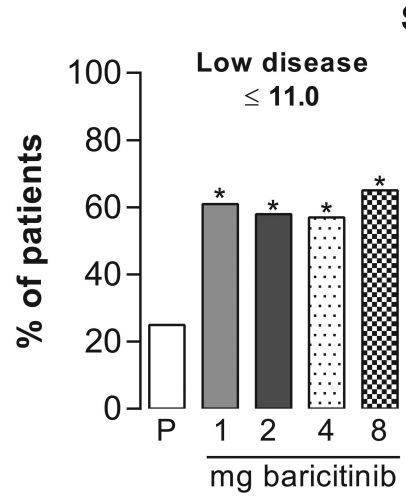

SDAI
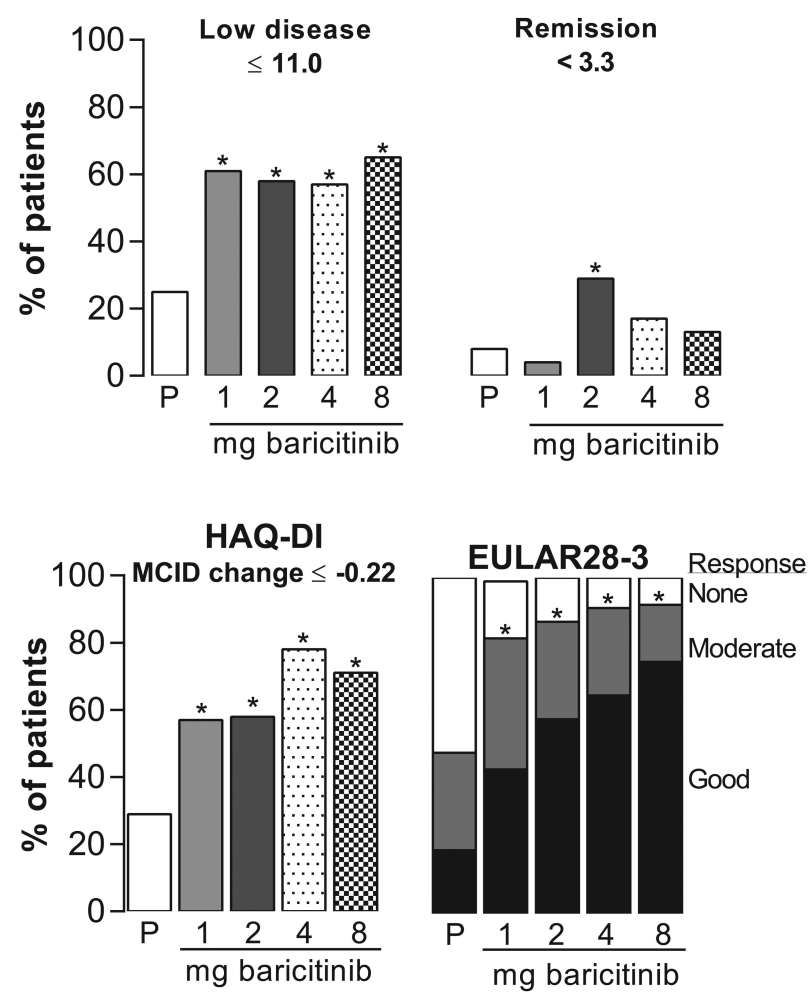

Figure 3. Additional efficacy outcomes for the 12-week study of Japanese patients with rheumatoid arthritis treated with baricitinib or placebo. Results at 12 weeks are presented. For DAS28-CRP, DAS28-ESR, SDAI, and HAQ-DI MCID, the proportion of patients with low disease, remission, or a clinically important change at 12 weeks of treatment is presented. For EULAR28-3, the proportion of patients with a good, moderate, or no response at 12 weeks of treatment is presented. Statistical comparisons were based on the Fisher's exact test of each dose versus placebo. ${ }^{*} \mathrm{p}<0.05$. DAS28: Disease Activity Score at 28 joints; CRP: C-reactive protein; ESR: erythrocyte sedimentation rate; SDAI: Simplified Disease Activity Index; HAQ-DI: Health Assessment Questionnaire-Disability Index; MCID: minimal clinically important difference; EULAR: European League Against Rheumatism; P: placebo.

JAK2/JAK2 homodimer ${ }^{23}$. Baricitinib at doses of $1 \mathrm{mg}, 2$ $\mathrm{mg}$, and $4 \mathrm{mg}$ did not affect hemoglobin concentrations or increase the percentage of patients experiencing a postbaseline increase in anemia based on CTCAE grade compared with placebo. However, an influence on erythropoietin signaling cannot be excluded at these doses because an increase in hemoglobin concentration may have been anticipated given the observed reduction in systemic inflammation. The 8-mg dose of baricitinib was associated with a larger decline in median hemoglobin concentration and with a higher percentage of patients experiencing a postbaseline increase in anemia based on CTCAE grade.

Larger decreases in median neutrophil count were observed for all baricitinib groups compared with placebo. However, the incidence of Grade 1 or higher neutropenia was infrequent and similar between placebo and baricitinib groups, indicating that the decreases in neutrophil counts occurred within the normal range. This decrease in neutrophil count may reflect a decline in systemic inflammation associated with baricitinib treatment.

Abnormally low baseline lymphocyte counts were frequent across baricitinib and placebo groups and may reflect treatment with MTX. Treatment with baricitinib at 4 $\mathrm{mg}$ and $8 \mathrm{mg}$ was associated with a higher percentage of patients experiencing a postbaseline increase in CTCAE grade for lymphopenia, although there was no change in median lymphocyte count over time for any group compared with placebo.

Treatment with baricitinib was also associated with increases in biochemical measures including serum creatinine, ALT, CPK, and LDL cholesterol. The etiology of the increase in creatinine is unclear, but may reflect inhibition of tubular secretion of creatinine, as has been postulated for other JAK inhibitors ${ }^{24}$. Increases in CPK were not associated with adverse events consistent with muscle injury or myositis. In addition, LDL cholesterol and HDL cholesterol increased in a dose-dependent fashion, which is consistent with studies of other therapies that inhibit JAK or IL-6 activity $25,26,27$. The longterm effect of baricitinib on lipid metabolism and the effect on the cardiovascular system needs careful evaluation in future studies. No patients discontinued the study because of a laboratory abnormality.

Personal non-commercial use only. The Journal of Rheumatology Copyright @ 2016 . All rights reserved. 
Table 2. Adverse events reported during the 12-week study of Japanese patients with rheumatoid arthritis treated with baricitinib or placebo. Values are $\mathrm{n}(\%)$.

\begin{tabular}{|c|c|c|c|c|c|}
\hline \multirow[t]{2}{*}{ Events } & \multirow[t]{2}{*}{ Placebo, $n=49$} & \multicolumn{4}{|c|}{ Baricitinib QD } \\
\hline & & $\begin{array}{l}1 \mathrm{mg} \\
\mathrm{n}=24\end{array}$ & $\begin{array}{l}2 \mathrm{mg} \\
\mathrm{n}=24\end{array}$ & $\begin{array}{l}4 \mathrm{mg} \\
\mathrm{n}=24\end{array}$ & $\begin{array}{l}8 \mathrm{mg}, \\
\mathrm{n}=24\end{array}$ \\
\hline TEAE & $26(53)$ & $11(46)$ & $12(50)$ & $13(54)$ & $18(75)$ \\
\hline SAE* & $1(2)$ & 0 & $1(4)$ & 0 & $1(4)$ \\
\hline $\begin{array}{l}\text { TEAE leading to discontinuation from } \\
\text { the study** }\end{array}$ & $1(2)$ & $1(4)$ & 0 & 0 & 0 \\
\hline TEAE related to infections $* * *$ & $11(22)$ & $6(25)$ & $4(17)$ & $7(29)$ & $5(21)$ \\
\hline Common adverse events ${ }^{\dagger}$ & & & & & \\
\hline Nasopharyngitis & $6(12)$ & $2(8)$ & $2(8)$ & $2(8)$ & $2(8)$ \\
\hline Abnormal hepatic function $\stackrel{\ddagger}{\ddagger}$ & 0 & 0 & $3(13)$ & 0 & $3(13)$ \\
\hline Blood creatine phosphokinase increased & $2(4)$ & 0 & 0 & $2(8)$ & $3(13)$ \\
\hline Abnormal liver function test & 0 & $1(4)$ & 0 & $2(8)$ & $2(8)$ \\
\hline Hypercholesterolemia & 0 & 0 & 0 & $1(4)$ & $3(13)$ \\
\hline Pharyngitis & $1(2)$ & $1(4)$ & $1(4)$ & 0 & $2(8)$ \\
\hline Hyperlipidemia & $1(2)$ & $1(4)$ & $1(4)$ & 0 & $1(4)$ \\
\hline Lymphopenia & $1(2)$ & 0 & $1(4)$ & $1(4)$ & $1(4)$ \\
\hline
\end{tabular}

* Placebo group: cholecystitis; 2-mg baricitinib group: acute pancreatitis; 8-mg baricitinib group: cataract.

** Placebo group: pollakiuria; 1-mg baricitinib group: herpes simplex. *** TEAE reported for the system organ class of infections and infestations. ${ }^{\dagger}$ Reported by at least 4 patients. $\stackrel{\ddagger}{*}$ For these cases, all reports were mild or moderate and recovered. QD: once daily; TEAE: treatment-emergent adverse event; SAE: serious adverse event.

Table 3. Median change from baseline to Week 12 in laboratory measures taken during the 12-week study of Japanese patients with rheumatoid arthritis treated with baricitinib or placebo. Values are median (minimum to maximum). Note that patient numbers varied from 48 to 49 for the placebo group, and from 22 to 24 for the baricitinib group.

\begin{tabular}{|c|c|c|c|c|c|}
\hline \multirow[t]{2}{*}{ Measures } & \multirow[t]{2}{*}{ Placebo } & \multicolumn{4}{|c|}{ Baricitinib QD } \\
\hline & & $1 \mathrm{mg}$ & $2 \mathrm{mg}$ & $4 \mathrm{mg}$ & $8 \mathrm{mg}$ \\
\hline Hemoglobin, mmol/l & $-0.125(-1.18$ to 1.06$)$ & 0.130 (-0.94 to 2.54$)$ & $-0.090(-1.42$ to 0.93$)$ & $0(-1.30$ to 0.50$)$ & $-0.190(-1.12$ to 1.06$)$ \\
\hline Lymphocytes, bill/1 & $0.030(-1.38$ to 0.73$)$ & 0.070 (-1.19 to 0.69$)$ & $0.075(-0.57$ to 0.76$)$ & $0.040(-0.50$ to 2.19$)$ & $-0.020(-1.07$ to 3.20$)$ \\
\hline Creatinine, $\mu \mathrm{mol} / 1$ & $0(-13$ to 10$)$ & $4.0 *(-3$ to 11$)$ & $3.0 *(-5$ to 13$)$ & $5.0 *(-2$ to 17$)$ & $5.0 *(-5$ to 23$)$ \\
\hline ALT, units/l & $1.0(-24$ to 37$)$ & $1.0(-22$ to 40$)$ & $2.5(-125$ to 24$)$ & $5.0 *(-41$ to 58$)$ & $3.0(-31$ to 83$)$ \\
\hline LDL cholesterol, mmol/1 & $-0.050(-0.72$ to 0.70$)$ & $0.130 *(-0.54$ to 0.78$)$ & $0.255^{*}(-1.19$ to 1.68$)$ & $0.310 *(-0.44$ to 1.17$)$ & $0.465 *(-2.23$ to 1.32$)$ \\
\hline CPK, units/l & $-2.0(-85$ to 55$)$ & $18.0 *(-123$ to 225$)$ & $30.0 *(-43$ to 611$)$ & $56.0 *(-10$ to 231$)$ & $69.0 *(13$ to 387$)$ \\
\hline
\end{tabular}

* Statistically significant difference in the mean change from baseline compared with placebo ( $<<0.05)$. QD: once daily; ALT: alanine aminotransferase; AST: aspartate aminotransferase; bill: billion; HDL: high-density lipoprotein; LDL: low-density lipoprotein; CPK, creatine phosphokinase.

Our study has a number of limitations. The number of patients in our study is relatively small, preventing robust evaluation of the influence of baseline patient characteristics or concomitant treatment on the efficacy and safety of baricitinib. The unbalanced randomization, coupled with the relatively short duration of treatment, limits the ability to estimate the incidence of infrequent adverse events that might be related to baricitinib treatment. In addition, the effect of baricitinib on the progression of structural joint damage was not evaluated.

Baricitinib in combination with MTX was efficacious and generally well tolerated over 12 weeks in Japanese patients with moderate to severe active RA. Although all doses of baricitinib were effective, the 4-mg and 8-mg doses were associated with earlier benefit and greater magnitude of improvement in some but not all disease activity measures compared with 1-mg and 2-mg doses. The 4-mg and 8-mg doses produced similar improvements in signs and symptoms of RA and patient function. However, the 8-mg dose was associated with a higher incidence of adverse events and laboratory abnormalities. Therefore, the 4-mg, and potentially the 2-mg, dose of baricitinib warrant further evaluation as a treatment for patients with moderate to severe active RA. Ongoing studies will further inform the benefit:risk profile of baricitinib in this common and disabling disease.

\section{ACKNOWLEDGMENT}

The authors are deeply grateful to the investigators and staff at all the study 
sites, listed as follows: National Nagasaki Medical Center, Nagasaki Medical Hospital of Rheumatology, Sagamihara National Hospital, Oribe Clinic Rheumatism and Medicine, Shinko Hospital, Kawasaki Municipal Kawasaki Hospital, Hokkaido Medical Center for Rheumatic Diseases, PS Clinic, Matsubara Mayflower Hospital, Taga General Hospital, National Tokyo Medical Center, Higashi-Hiroshima Memorial Hospital, National Hospital Organization Osaka Minami Medical Center, Izumihara Rheumatism Internal Medicine Clinic, National Hospital Organization Kyushu Medical Center, Shono Rheumatism Clinic, Sasebo Chuo Hospital, Red Cross Okayama Hospital, Honjo Rheumatism Clinic, National Chiba-East Hospital, Matsubara Clinic, Hiroshima Rheumatology Clinic, Hikarichuo Clinic, and the Hiroshima Red Cross/Atom Bomb Hospital.

\section{ONLINE SUPPLEMENT}

Supplementary data for this article are available online at jrheum.org.

\section{REFERENCES}

1. McInnes IB, Schett G. The pathogenesis of rheumatoid arthritis. N Engl J Med 2011;365:2205-19.

2. Gordon RA, Grigoriev G, Lee A, Kalliolias GD, Ivashkiv LB. The interferon signature and STAT1 expression in rheumatoid arthritis synovial fluid macrophages are induced by tumor necrosis factor $\alpha$ and counter-regulated by the synovial fluid microenvironment. Arthritis Rheum 2012;64:3119-28.

3. O'Shea JJ, Kontzias A, Yamaoka K, Tanaka Y, Laurence A. Janus kinase inhibitors in autoimmune diseases. Ann Rheum Dis 2013;72 Suppl 2:ii111-5.

4. Leonard WJ, O'Shea JJ. Jaks and STATs: biological implications. Annu Rev Immunol 1998;16:293-322.

5. Kubo S, Yamaoka K, Kondo M, Yamagata K, Zhao J, Iwata S, et al. The JAK inhibitor, tofacitinib, reduces the T cell stimulatory capacity of human monocyte-derived dendritic cells. Ann Rheum Dis 2014;73:2192-8.

6. Fridman JS, Scherle PA, Collins R, Burn TC, Li Y, Li J, et al. Selective inhibition of JAK1 and JAK2 is efficacious in rodent models of arthritis: preclinical characterization of INCB028050. J Immunol 2010;184:5298-307.

7. Smolen JS, Landewé R, Breedveld FC, Buch M, Burmester G, Dougados M, et al. EULAR recommendations for the management of rheumatoid arthritis with synthetic and biological disease-modifying antirheumatic drugs: 2013 update. Ann Rheum Dis 2014;73:492-509.

8. Norman P. Selective JAK inhibitors in development for rheumatoid arthritis. Expert Opin Investig Drugs 2014;23:1067-77.

9. Keystone EC, Taylor PC, Drescher E, Schlichting DE, Beattie SD, Berclaz PY, et al. Safety and efficacy of baricitinib at 24 weeks in patients with rheumatoid arthritis who have had an inadequate response to methotrexate. Ann Rheum Dis 2015;74:333-40.

10. Keystone EC, Taylor PC, Genovese MC, Schlichting DE, De La Torre I, Beattie SD, et al. Safety and efficacy of baricitinib through 128 weeks in an open-label, long-term extension study in patients with rheumatoid arthritis. [Internet. Accessed November 27, 2015.] Available from: acrabstracts.org/abstract/safety-and-efficacy-ofbaricitinib-through-128-weeks-in-an-open-label-long-termextension-study-in-patients-with-rheumatoid-arthritis

11. Taylor P, Genovese MC, Keystone E, Schlichting D, Beattie S, Macias W. Baricitinib, an oral Janus kinase inhibitor, in the treatment of rheumatoid arthritis: safety and efficacy in open-label, long-term extension study. Ann Rheum Dis 2013;72 Suppl 3:65

12. Kochi Y, Suzuki A, Yamada R, Yamamoto K. Ethnogenetic heterogeneity of rheumatoid arthritis-implications for pathogenesis. Nat Rev Rheumatol 2010;6:290-5.

13. Alamanos Y, Drosos AA. Epidemiology of adult rheumatoid arthritis. Autoimmun Rev 2005;4:130-6.
14. Ogasawara M, Tamura N, Onuma S, Kusaoi M, Sekiya F, Matsudaira R, et al. Observational cross-sectional study revealing less aggressive treatment in Japanese elderly than nonelderly patients with rheumatoid arthritis. J Clin Rheumatol 2010;16:370-4.

15. Yamanaka H, Tanaka Y, Inoue E, Hoshi D, Momohara S, Hanami K, et al. Efficacy and tolerability of tocilizumab in rheumatoid arthritis patients seen in daily clinical practice in Japan: results from a retrospective study (REACTION study). Mod Rheumatol 2011;21:122-33.

16. Harrold LR, Harrington JT, Curtis JR, Furst DE, Bentley MJ, Shan $\mathrm{Y}$, et al. Prescribing practices in a US cohort of rheumatoid arthritis patients before and after publication of the American College of Rheumatology treatment recommendations. Arthritis Rheum 2012;64:630-8.

17. Hochberg MC, Chang RW, Dwosh I, Lindsey S, Pincus T, Wolfe F. The American College of Rheumatology 1991 revised criteria for the classification of global functional status in rheumatoid arthritis. Arthritis Rheum 1992;35:498-502.

18. Aletaha D, Landewé R, Karonitsch T, Bathon J, Boers M, Bombardier C, et al. Reporting disease activity in clinical trials of patients with rheumatoid arthritis: EULAR/ACR collaborative recommendations. Arthritis Rheum 2008;59:1371-7.

19. Smolen JS, Breedveld FC, Eberl G, Jones I, Leeming M, Wylie GL, et al. Validity and reliability of the twenty-eight-joint count for the assessment of rheumatoid arthritis activity. Arthritis Rheum 1995;38:38-43.

20. Villaverde V, Balsa A, Cantalejo M, Fernández-Prada M, Madero MR, Munoz-Fernandez S, et al. Activity indices in rheumatoid arthritis. J Rheumatol 2000;27:2576-81.

21. Greenwald MW, Fidelus-Gort R, Levy R, Liang J, Vaddi K, Williams WV, et al. A randomized dose-ranging, placebo-controlled study of INCB028050, a selective JAK1 and JAK2 inhibitor in subjects with active rheumatoid arthritis. Arthritis Rheum 2010;62 Suppl 10:2172.

22. Kremer JM, Cohen S, Wilkinson BE, Connell CA, French JL, Gomez-Reino J, et al. A phase IIb dose-ranging study of the oral JAK inhibitor tofacitinib $(\mathrm{CP}-690,550)$ versus placebo in combination with background methotrexate in patients with active rheumatoid arthritis and an inadequate response to methotrexate alone. Arthritis Rheum 2012;64:970-81.

23. O'Shea JJ, Schwartz DM, Villarino AV, Gadina M, McInnes IB, Laurence A. The JAK-STAT pathway: impact on human disease and therapeutic intervention. Ann Rev Med 2015;66:311-28.

24. Kremer JM, Bloom BJ, Breedveld FC, Coombs JH, Fletcher MP, Gruben D, et al. The safety and efficacy of a JAK inhibitor in patients with active rheumatoid arthritis: results of a double-blind, placebo-controlled phase IIa trial of three dosage levels of CP-690,550 versus placebo. Arthritis Rheum 2009;60:1895-905.

25. Kawashiri SY, Kawakami A, Yamasaki S, Imazato T, Iwamoto N, Fujikawa K, et al. Effects of the anti-interleukin-6 receptor antibody, tocilizumab, on serum lipid levels in patients with rheumatoid arthritis. Rheumatol Int 2011;31:451-6.

26. Nishimoto N, Yoshizaki K, Maeda K, Kuritani T, Deguchi H, Sato $\mathrm{B}$, et al. Toxicity, pharmacokinetics, and dose-finding study of repetitive treatment with the humanized anti-interleukin 6 receptor antibody MRA in rheumatoid arthritis. Phase I/II clinical study. J Rheumatol 2003;30:1426-35.

27. Souto A, Salgado E, Maneiro JR, Mera A, Carmona L, Gómez-Reino JJ. Lipid profile changes in patients with chronic inflammatory arthritis treated with biologic agents and tofacitinib in randomized clinical trials: a systematic review and meta-analysis. Arthritis Rheumatol 2015;67:117-27. 


\section{Correction}

Efficacy and Safety of Baricitinib in Japanese Patients with Active Rheumatoid Arthritis Receiving Background Methotrexate Therapy: A 12-week, Double-blind, Randomized Placebo-controlled Study

Tanaka Y, Emoto K, Cai Z, Aoki T, Schlichting D, Rooney $\mathrm{T}$, et al. Efficacy and safety of baricitinib in Japanese patients with active rheumatoid arthritis receiving background methotrexate therapy: a 12-week, double-blind, randomized placebo-controlled study. J Rheumatol 2016;43:504-11. In the Materials and Methods section, fifth paragraph, this sentence should read as follows:

A minimal clinically important difference (MCID) in HAQ-DI was defined as a change from baseline of $\leq-0.22$. [Not $\geq-0.22$.]

We regret the error.

doi:10.3899/jrheum.150613.C1 